\title{
APPROXIMATION SOLUTIONS OF QUASI-EQUILIBRIUM PROBLEMS IN BANACH SPACES
}

\author{
MEHDI MOHAMMADI AND G. ZAMANI ESKANDANI
}

Received 16 October, 2019

\begin{abstract}
In this paper, we study to approximate solutions of quasi-equilibrium problems in Banach spaces, which generalizes equilibrium problems and quasi-variational inequalities. In fact, we prove weak convergence of the sequence generated by the proximal point method to a solution of the quasi-equilibrium problem. Then we show that the generated sequence converges strongly to a solution of the quasi-equilibrium problem.
\end{abstract}

2010 Mathematics Subject Classification: 90C33; 74G10

Keywords: demiclosed, quasi-equilibrium problem, quasi $\phi$-nonexpansive mapping, strong convergence, weak convergence

\section{INTRODUCTION}

Let $E$ be a real Banach space and $C \subset E$ be a nonempty, closed and convex set, and $K: C \rightarrow 2^{C}$ be a multivalued mapping such that for all $x \in C, K(x)$ is a nonempty, closed and convex subset of $C$. Suppose that $f: E \times E \rightarrow \mathbb{R}$ is a bifunction. The quasi-equilibrium problem $(\mathrm{QEP}(f, K))$ is to find $x^{*} \in K\left(x^{*}\right)$ such that

$$
f\left(x^{*}, y\right) \geq 0, \quad \forall y \in K\left(x^{*}\right) .
$$

The set of all solutions of $\operatorname{QEP}(f, K)$ is denoted by $S(f, K)$. Also, the set of all fixed points of $K$ is denoted by $\operatorname{Fix}(K)$. The associated Minty quasi-equilibrium problem is to find $x^{*} \in K\left(x^{*}\right)$ such that $f\left(y, x^{*}\right) \leq 0$ for all $y \in K\left(x^{*}\right)$. When $K(x)=C$ for all $x \in C$, the quasi-equilibrium problem $\operatorname{QEP}(f, K)$ becomes a classical equilibrium problem $\operatorname{EP}(f, C)$, also the associated Minty quasi-equilibrium problem becomes a classical Minty equilibrium problem (see [2] and [20]).

Equilibrium problems extend and unify many problems in optimization, variational inequalities, fixed point theory, complementarity problems, Nash equilibria and many other problems in nonlinear analysis (see [7], [9] and [10]).

Recently many researchers have studied approximation of solutions of an equilibrium problem (see, e.g. [6], [9], [10], [15], [16], [18] and references therein). Equilibrium problems with monotone and pseudo-monotone bifunctions have been studied extensively in Hilbert, Banach as well as in topological vector spaces by many 
authors (e.g. [3], [4], [5], [7], [10], [11], [14], [13]). Also, the quasi-equilibrium problems have been studied in [2] and [20]. Our aim in this paper is to approximate a solution of quasi-equilibrium problems. This type of problems arises, for example, in the electricity market (see [17]).

This paper is organized as follows. In Section 2, we introduce some preliminary material related to the geometry of Banach spaces. In Section 3, we prove weak convergence of the sequence generated by proximal point method to a solution of quasi-equilibrium problems. In Section 4, we study strong convergence of the generated sequence to a solution of the problems by imposing some additional conditions.

\section{PRELIMINARIES}

Let $E$ be a real Banach space with norm $\|$.$\| and E^{*}$ denote the dual of $E$. We denote the value of $v \in E^{*}$ at $x \in E$ by $\langle x, v\rangle$. The duality mapping $J$ from $E$ into $2^{E^{*}}$ is defined by:

$$
J x=\left\{v \in E^{*}:\langle x, v\rangle=\|x\|^{2}=\|v\|^{2}\right\},
$$

for $x \in E$. When $E$ is a smooth Banach space, we can use the following function studied by Alber [1], Kamimura and Takahashi [12] and Reich [19]:

$$
\phi(x, y)=\|x\|^{2}-2\langle x, J y\rangle+\|y\|^{2},
$$

for all $x, y \in E$. It is obvious from the definition of $\phi$ that

$$
0 \leq(\|x\|-\|y\|)^{2} \leq \phi(x, y),
$$

for all $x, y \in E$. Note that if $E$ is a Hilbert space, then $\phi(x, y)=\|x-y\|^{2}$.

Proposition 1 ([12]). Let $E$ be a uniformly convex and smooth Banach space and let $\left\{x_{n}\right\}$ and $\left\{y_{n}\right\}$ be two sequences of $E$. If $\lim _{n \rightarrow \infty} \phi\left(x_{n}, y_{n}\right)=0$ and either $\left\{x_{n}\right\}$ or $\left\{y_{n}\right\}$ is bounded, then $\lim _{n \rightarrow \infty}\left\|x_{n}-y_{n}\right\|=0$.

Proposition 2 ([12]). Let $E$ be a reflexive, strictly convex and smooth Banach space, and let $C$ be a nonempty closed convex subset of $E$ and $x \in E$. Then there exists a unique element $\bar{x} \in C$ such that $\phi(\bar{x}, x)=\inf \{\phi(z, x): z \in C\}$.

Regarding Proposition 2, we denote the unique element $\bar{x} \in C$ by $P_{C}(x)$, where the mapping $P_{C}$ is called the generalized projection from $E$ onto $C$. It is obvious that in Hilbert spaces, $P_{C}$ is coincident with the metric projection from $E$ onto $C$.

Proposition 3 ([12]). Let $E$ be a smooth Banach space, $C$ be a convex subset of $E, x \in E$ and $\bar{x} \in C$. Then $\phi(\bar{x}, x)=\inf \{\phi(z, x): z \in C\}$ if and only if

$$
\langle z-\bar{x}, J x-J \bar{x}\rangle \leq 0, \forall z \in C .
$$

Throughout this paper we assume that $E$ is a real Banach space which is uniformly convex and uniformly smooth, and $C \subset E$ is nonempty closed and convex unless otherwise specified. When $\left\{x_{n}\right\}$ is a sequence in $E$, we denote strong convergence of $\left\{x_{n}\right\}$ to $x \in E$ by $x_{n} \rightarrow x$ and weak convergence by $x_{n} \rightarrow x$. 
Definition 1. The mapping $T: C \rightarrow C$ is called quasi $\phi$-nonexpansive whenever $\operatorname{Fix}(T) \neq \varnothing$ and $\phi(p, T x) \leq \phi(p, x)$ for all $(p, x) \in \operatorname{Fix}(T) \times C$.

The following definitions are adapted from [20].

Definition 2. Suppose that $K: C \rightarrow 2^{C}$ is a multivalued mapping such that for every $x \in C, K(x)$ is nonempty, closed and convex. $K$ is called quasi $\phi$-nonexpansive whenever the mapping $T(\cdot)=P_{K(\cdot)}(\cdot)$ is quasi $\phi$-nonexpansive where $P$ is the generalized projection.

Definition 3. We say that $K: C \rightarrow 2^{C}$ is weak lower semicontinuous at each $\bar{x} \in C$, whenever we have $\left\{x_{k}\right\} \subset C$ and $x_{k} \rightarrow \bar{x}$, then for any $\bar{y} \in K(\bar{x})$, there is a sequence $\left\{y_{k}\right\}$ with $y_{k} \in K\left(x_{k}\right)$ for every $\mathrm{k}$, such that $y_{k} \rightarrow \bar{y}$ as $k \rightarrow \infty$.

Definition 4. We say that $K: C \rightarrow 2^{C}$ is demiclosed, whenever we have $x_{k} \rightarrow \bar{x}$ and $\lim _{k \rightarrow \infty} d\left(x_{k}, K\left(x_{k}\right)\right)=0$, then $\bar{x} \in \operatorname{Fix}(K)$.

It is well known that if $T$ is a quasi $\phi$-nonexpansive mapping, then $\operatorname{Fix}(T)$ is convex. Also, if $T$ is demiclosed then $\operatorname{Fix}(T)$ is closed.

A bifunction $f$ is called "monotone" if $f(x, y)+f(y, x) \leq 0$ for all $x, y \in E$, and "pseudo-monotone" if for any pair $x, y \in E, f(x, y) \geq 0$ implies $f(y, x) \leq 0$.

We introduce some conditions on the bifunction $f$ and the multivalued mapping $K$ where $K$ is quasi $\phi$-nonexpansive, which are needed in the convergence analysis.

B1: $f(x, x)=0$ for all $x \in E$.

B2: $f(\cdot, \cdot): E \times E \rightarrow \mathbb{R}$ is continuous.

B3: $f(x, \cdot): E \rightarrow \mathbb{R}$ is convex for all $x \in E$.

B4: There exists $\theta \geq 0$ such that $f(x, y)+f(y, x) \leq \frac{\theta}{2}(\phi(x, y)+\phi(y, x))$, for all $x, y \in E$ ( $f$ is called $\theta$-undermonotone).

B5: If $\left\{x_{k}\right\}$ and $\left\{y_{k}\right\}$ are two arbitrary sequences in $C$ such that $x_{k} \rightarrow \bar{x}, y_{k} \rightarrow \bar{y}$ and also we have $\limsup _{k \rightarrow \infty} f\left(x_{k}, y_{k}\right) \geq 0$, then $f(\bar{x}, \bar{y}) \geq 0$.

B6: $K: C \rightarrow 2^{C}$ is demiclosed and lower semicontinuous at each $x \in C$.

Finally, in order to well definedness and boundedness of the generated sequences by our algorithm in this paper, we assume that

$$
S_{*}=\left\{x \in \cap_{z \in C} K(z): f(y, x) \leq 0, \quad \forall y \in \cup_{z \in C} K(z)\right\} \neq \varnothing,
$$

Note that under B1-B3, $S_{*}$ is closed and convex.

\section{Proximal Point Method AND WEAK CONVERGENCE}

In this section, we study the weak convergence of the sequence generated by the following algorithm. We will assume that $E$ is a uniformly smooth and uniformly convex Banach space, $C \subseteq E$ is nonempty closed and convex. Let $K: C \rightarrow 2^{C}$ be a multivalued quasi $\phi$-nonexpansive mapping, and let $f: E \times E \rightarrow \mathbb{R}$ be a bifunction, $S_{*} \neq \varnothing$, and the assumptions B1-B6 are satisfied. 


\section{Algorithm 1.}

1. Initialization: Take $x_{0} \in E$ and consider $\beta>0$ satisfying $\theta<\beta$ where $\theta$ is the undermonotonicity constant of $f$, and a sequence $\left\{\lambda_{k}\right\} \subset(\theta, \beta]$.

2. Iterative step: Given $x_{k}$, define

$$
y_{k}=P_{K\left(x_{k}\right)}\left(x_{k}\right) .
$$

Find $z_{k}$ such that:

$$
f\left(z_{k}, y\right)+\lambda_{k}\left\langle y-z_{k}, J z_{k}-J y_{k}\right\rangle \geq 0, \quad \forall y \in K\left(x_{k}\right),
$$

if $z_{k}=x_{k}$ stop. Otherwise, define

$$
\begin{gathered}
H_{k}=\left\{y \in E:\left\langle y-z_{k}, J z_{k}-J y_{k}\right\rangle \geq 0\right\}, \\
C_{k}=C \cap\left(\cap_{i=0}^{k} H_{i}\right), \\
x_{k+1}=P_{C_{k}}\left(z_{k}\right) .
\end{gathered}
$$

The following proposition ensures the existence of the sequence $\left\{z_{k}\right\}$ generated by (3.2). It is easy to see that if $S_{*} \neq \varnothing$, then the sequences $\left\{x_{k}\right\}$ and $\left\{y_{k}\right\}$ are well defined.

Proposition 4 ([8]). If $f$ satisfies $B 1-B 4$ and $\lambda>\theta$, then $E P(\tilde{f}, C)$ has a unique solution where $\bar{x} \in E$ and $\tilde{f}(x, y)=f(x, y)+\lambda\langle y-x, J x-J \bar{x}\rangle$.

Proposition 5. If Algorithm 1 stops at the $k$-th iteration, then $z_{k}$ is a solution of $Q E P(f, K)$.

Proof. If $z_{k}=x_{k}$, then $z_{k}=y_{k}$ by (3.1). Now the result follows from (3.2).

Lemma 1. Suppose that $\left\{x_{k}\right\},\left\{y_{k}\right\}$ and $\left\{z_{k}\right\}$ are the sequences generated by the algorithm. If $S_{*} \neq \varnothing$, then $\lim _{k \rightarrow \infty} \phi\left(x^{*}, x_{k}\right)$ exists for all $x^{*} \in S_{*}$. Also $\left\{x_{k}\right\}$ is bounded and subsequently the sequences $\left\{y_{k}\right\}$ and $\left\{z_{k}\right\}$ are bounded and

$$
\lim _{k \rightarrow \infty}\left\|x_{k+1}-z_{k}\right\|=\lim _{k \rightarrow \infty}\left\|x_{k}-y_{k}\right\|=\lim _{k \rightarrow \infty}\left\|y_{k}-z_{k}\right\|=0 .
$$

Proof. Note that since $y_{k}=P_{K\left(x_{k}\right)}\left(x_{k}\right)$ and $P_{K(\cdot)}(\cdot)$ is a quasi $\phi$-nonexpansive mapping, we have

$$
\left\langle x^{*}-y_{k}, J x_{k}-J y_{k}\right\rangle \leq 0,
$$

where $x^{*} \in S_{*}$. Therefore, we get

$$
\phi\left(x^{*}, y_{k}\right)+\phi\left(y_{k}, x_{k}\right)-\phi\left(x^{*}, x_{k}\right) \leq 0 .
$$

On the other hand, since $f\left(z_{k}, x^{*}\right) \leq 0$, hence (3.2) implies that $x^{*} \in H_{k}$ for all $k$. Therefore we have

which implies that

$$
\left\langle z_{k}-x^{*}, J z_{k}-J y_{k}\right\rangle \leq 0,
$$

$$
\phi\left(x^{*}, z_{k}\right)+\phi\left(z_{k}, y_{k}\right)-\phi\left(x^{*}, y_{k}\right) \leq 0 \text {. }
$$


Since $x_{k+1}=P_{C_{k}}\left(z_{k}\right)$, therefore we have

$$
\left\langle x^{*}-x_{k+1}, J z_{k}-J x_{k+1}\right\rangle \leq 0 .
$$

By proposition 3 which implies that

$$
\phi\left(x^{*}, x_{k+1}\right)+\phi\left(x_{k+1}, z_{k}\right)-\phi\left(x^{*}, z_{k}\right) \leq 0 .
$$

Hence (3.7), (3.9) and (3.11) imply that

$$
\begin{aligned}
\phi\left(x^{*}, x_{k+1}\right) & \leq \phi\left(x^{*}, x_{k+1}\right)+\phi\left(x_{k+1}, z_{k}\right) \\
& \leq \phi\left(x^{*}, z_{k}\right) \leq \phi\left(x^{*}, z_{k}\right)+\phi\left(z_{k}, y_{k}\right) \leq \phi\left(x^{*}, y_{k}\right) \\
& \leq \phi\left(x^{*}, y_{k}\right)+\phi\left(y_{k}, x_{k}\right) \leq \phi\left(x^{*}, x_{k}\right) .
\end{aligned}
$$

Therefore $\lim _{k \rightarrow \infty} \phi\left(x^{*}, x_{k}\right)$ exists. Also $\left\{x_{k}\right\}$ is bounded by (2.2). Passing to the limit in the above inequality as $k \rightarrow \infty$, one gets

$$
\lim _{k \rightarrow \infty} \phi\left(x_{k+1}, z_{k}\right)=\lim _{k \rightarrow \infty} \phi\left(z_{k}, y_{k}\right)=\lim _{k \rightarrow \infty} \phi\left(y_{k}, x_{k}\right)=0 .
$$

Now, by Proposition 1, we have

$$
\lim _{k \rightarrow \infty}\left\|x_{k+1}-z_{k}\right\|=\lim _{k \rightarrow \infty}\left\|z_{k}-y_{k}\right\|=\lim _{k \rightarrow \infty}\left\|y_{k}-x_{k}\right\|=0 .
$$

Proposition 6. Suppose that $f$ is a bifunction, $K$ is a multivalued quasi $\phi$-nonexpansive mapping and the conditions B1-B6 are satisfied. If there exists a subsequence $\left\{x_{k_{n}}\right\}$ of $\left\{x_{k}\right\}$ such that $x_{k_{n}} \rightarrow p$, then

i) $p \in \operatorname{Fix}(K)$,

ii) $\lim _{k \rightarrow \infty} \phi\left(q, x_{k}\right)$ exists.

Proof. i) Let $\left\{x_{k_{n}}\right\}$ be a subsequence of $\left\{x_{k}\right\}$ such that $x_{k_{n}} \rightarrow p$. Note that $\lim _{n \rightarrow \infty}\left\|x_{k_{n}}-y_{k_{n}}\right\|=0$ by Lemma 1 , where for each $n, y_{k_{n}}$ is the generalized projection of $x_{k_{n}}$ onto $K\left(x_{k_{n}}\right)$. Therefore we have $\lim _{n \rightarrow \infty} d\left(x_{k_{n}}, K\left(x_{k_{n}}\right)\right)=0$. Since $K$ is demiclosed, thus $p \in K(p)$.

ii) First we show that $p \in \cap_{k=0}^{\infty} C_{k}$, it is sufficient to prove that $p \in C_{k}$ for all integer $k$. Note that the sequence $\left\{C_{k}\right\}$ is nonincreasing, let $N$ be a fixed integer, hence there is $j>N$ such that for all $n \geq j$, we have

$$
x_{k_{n}+1} \in C_{k_{n}} \subseteq C_{N},
$$

where $x_{k_{n}+1}=P_{C_{k_{n}}}\left(z_{k_{n}}\right)$. Since $x_{k_{n}} \rightarrow p$, we have $x_{k_{n}+1} \rightarrow p$ by Lemma 1. Consequently, $C_{N}$ is closed and $p \in C_{N}$, the weak limit point of $\left\{x_{k_{n}}\right\}$. Since $N$ is arbitrary, we obtain that

$$
p \in \cap_{k=0}^{\infty} C_{k} \text {. }
$$

It follows from (i) that $p \in K(p)$. Since $y_{k}=P_{K\left(x_{k}\right)}\left(x_{k}\right)$ and $P_{K(\cdot)}(\cdot)$ is a quasi $\phi$ nonexpansive mapping, we have

$$
\phi\left(p, y_{k}\right) \leq \phi\left(p, x_{k}\right)
$$


On the other hand, since $p \in H_{k}$ for all $k$, we have $\left\langle z_{k}-p, J z_{k}-J y_{k}\right\rangle \leq 0$, which implies that

$$
\phi\left(p, z_{k}\right)+\phi\left(z_{k}, y_{k}\right)-\phi\left(p, y_{k}\right) \leq 0 .
$$

Since $x_{k+1}=P_{C_{k}}\left(z_{k}\right)$, we have $\left\langle p-x_{k+1}, J z_{k}-J x_{k+1}\right\rangle \leq 0$ by Proposition 3, which implies that

$$
\phi\left(p, x_{k+1}\right)+\phi\left(x_{k+1}, z_{k}\right)-\phi\left(p, z_{k}\right) \leq 0 .
$$

Since $\phi$ is non-negative by (2.2), hence by using (3.13), (3.14) and (3.15), we get $\phi\left(p, x_{k+1}\right) \leq \phi\left(p, x_{k}\right)$. This implies that $\lim _{k \rightarrow \infty} \phi\left(p, x_{k}\right)$ exists.

In order to prove the uniqueness of the weak limit point of the sequence in the following theorem, we need the following condition on Banach space $E$, i.e. we assume that if $\left\{u_{k}\right\}$ and $\left\{v_{k}\right\}$ are arbitrary sequences in $K$ that converge weakly to $u$ and $v$, respectively, and $u \neq v$, then

$$
\liminf _{k \rightarrow \infty}\left|\left\langle u-v, J u_{k}-J v_{k}\right\rangle\right|>0 .
$$

For non-Hilbertian examples of such spaces, we can consider $\ell^{p}$ spaces for $1<p<$ $\infty$ that satisfy in the above condition. It is valuable to mention that we need this condition on $E$ just in the following theorem and we will never use it in the sequel, i.e. when we prove the strong convergence theorems in this paper we do not need the above condition.

Theorem 1. Suppose that $f$ is a bifunction, $K$ is a multivalued quasi $\phi$-nonexpansive mapping and the conditions B1-B6 are satisfied. If $S_{*} \neq \varnothing$ and the sequence $\left\{x_{k}\right\}$ generated by Algorithm 1, then

i) all cluster points of $\left\{x_{k}\right\}$ solve the problem,

ii) if in addition either $E$ satisfies (3.16) or the problem has a unique solution, then the whole sequence $\left\{x_{k}\right\}$ is weakly convergent to an element of $S(f, K)$.

Proof. Let $x_{k_{n}} \rightarrow \bar{x}$, therefore it is enough to show that $\bar{x} \in S(f, K)$. From Lemma 1 , we have

$$
\lim _{n \rightarrow \infty}\left\|z_{k_{n}}-y_{k_{n}}\right\|=0 \text {. }
$$

Now, uniform smoothness of $E$ implies uniform (norm-to-norm) continuity of $J$ on each bounded set of $E$. Therefore, we get from (3.17) that

$$
\lim _{n \rightarrow \infty}\left\|J z_{k_{n}}-J y_{k_{n}}\right\|=0 \text {. }
$$

On the other hand, since $x_{k_{n}} \rightarrow \bar{x}$, we have $z_{k_{n}} \rightarrow \bar{x}$ by Lemma 1 . Now, take any $y \in K(\bar{x})$, since $K$ is lower semicontinuous at $\bar{x} \in C$, therefore there is a sequence $\left\{\xi_{k_{n}}\right\}$ such that $\xi_{k_{n}} \in K\left(x_{k_{n}}\right)$ and $\xi_{k_{n}} \rightarrow y$. By (3.2), we have

$$
f\left(z_{k_{n}}, \xi_{k_{n}}\right)+\lambda_{k_{n}}\left\langle\xi_{k_{n}}-z_{k_{n}}, J z_{k_{n}}-J y_{k_{n}}\right\rangle \geq 0,
$$

which implies that

$$
f\left(z_{k_{n}}, \xi_{k_{n}}\right)+\lambda_{k_{n}}\left\|\xi_{k_{n}}-z_{k_{n}}\right\|\left\|J z_{k_{n}}-J y_{k_{n}}\right\| \geq 0 .
$$


We use (3.18) and (3.19) together with the boundedness of $\left\{\lambda_{k_{n}}\right\}$ and $\left\{\xi_{k_{n}}\right\}$ in order to obtain $\liminf _{n \rightarrow \infty} f\left(z_{k_{n}}, \xi_{k_{n}}\right) \geq 0$. Now B5 implies that $f(\bar{x}, y) \geq 0$. Since $y \in K(\bar{x})$ is arbitrary, hence $\bar{x} \in S(f, K)$.

ii) When the problem has a unique solution, the result is clear. Therefore we must show that there exists only one weak limit point of $\left\{x_{k}\right\}$ whenever $E$ satisfies (3.16). Suppose that $p$ is an other weak limit point of $\left\{x_{k}\right\}$, then there exists subsequence $\left\{x_{k_{j}}\right\}$ such that $x_{k_{j}} \rightarrow p$. We have already proved that $p$ is the element of $S(f, K)$. Using Proposition 6, we get $\lim _{k \rightarrow \infty} \phi\left(p, x_{k}\right)$ and $\lim _{k \rightarrow \infty} \phi\left(\bar{x}, x_{k}\right)$ exist. Note that

$$
\begin{aligned}
2\left\langle\bar{x}-p, J x_{k_{n}}-J x_{k_{j}}\right\rangle & =2\left\langle\bar{x}, J x_{k_{n}}\right\rangle-2\left\langle p, J x_{k_{n}}\right\rangle-2\left\langle\bar{x}, J x_{k_{j}}\right\rangle+2\left\langle p, J x_{k_{j}}\right\rangle \\
& =-\phi\left(\bar{x}, x_{k_{n}}\right)+\phi\left(p, x_{k_{n}}\right)+\phi\left(\bar{x}, x_{k_{j}}\right)-\phi\left(p, x_{k_{j}}\right)
\end{aligned}
$$

(In the second equality the relation (2.1) is used). Taking limit when $n \rightarrow \infty$ and then when $j \rightarrow \infty$, we obtain $p=\bar{x}$, i.e. $\left\{x_{k}\right\}$ converges weakly to a point of $S(f, K)$.

Example 1. Suppose that $C=\left\{X=\left[\begin{array}{c}x_{1} \\ \vdots \\ x_{n}\end{array}\right] ; a_{i} \leq x_{i} \leq b_{i}, \forall i=1, \ldots, n\right\}$ where $a_{i}$ and $b_{i}$ are real numbers, and the bifunction $f: C \times C \rightarrow \mathbb{R}$ is defined by $f(X, Y)=$ $X^{t} X-X^{t} Y$ where $X^{t}$ denotes the transpose of the matrix $X$. Note that the conditions B1-B5 are satisfied. Now we define the multivalued mapping $K: C \rightarrow 2^{C}$ by

$$
K(X)=\left\{Z \in C: z_{1}+\cdots+z_{n} \geq \max \left\{x_{1}+\ldots+x_{n}, \beta\right\}\right\},
$$

where $\beta$ is a constant number such that $\beta \leq \sum_{i=1}^{n} b_{i}$. Then, for each $x \in C, K(x)$ is a nonempty, closed and convex subset of $C$. It is also easy to see that $K$ is demiclosed and lower semicontinuous at each $x \in C$, i.e. the condition B6 is satisfied. Since $\left[b_{1}, \ldots, b_{n}\right]^{t} \in S_{*}$, it is easy to see that the assumptions of Theorem 1 are satisfied. Therefore we can use Theorem 1, i.e. if the sequence $\left\{x_{k}\right\}$ is generated by Algorithm 1 , it converges to an element of $S(f, K)$.

\section{Strong CONVERGENCE}

In this section, we study the strong convergence of the sequence generated by Algorithm 1 to an element of $S(f, K)$ with some additional assumptions on the problem.

Definition 5. A bifunction $f$ is called strongly monotone, if there exists $\alpha>0$ such that $f(x, y)+f(y, x) \leq-\alpha\|x-y\|^{2}$, for all $x, y \in E$.

Also, a bifunction $f$ is called strongly pseudo-monotone, if there exists $\beta>0$ such that whenever $f(x, y) \geq 0$, then $f(y, x) \leq-\beta\|x-y\|^{2}$, for all $x, y \in E$.

Definition 6. A function $h: E \rightarrow \mathbb{R}$ is called strongly convex, whenever for each pair $x, y \in E$ and each $\lambda \in[0,1]$, we have

$$
h(\lambda x+(1-\lambda) y) \leq \lambda h(x)+(1-\lambda) h(y)-\lambda(1-\lambda)\|x-y\|^{2} .
$$

We say that $h$ is strongly concave whenever $-h$ is strongly convex. 
Example 2. Suppose that $g: E \times E \rightarrow \mathbb{R}$ is monotone. If $\alpha: E \times E \rightarrow \mathbb{R}^{+}$, then $f(x, y):=\alpha(x, y) g(x, y)$ is pseudo-monotone but it is not necessarily monotone. Now if $\alpha(x, y) \geq c>0$ and $g$ is strongly monotone, then $f(x, y)=\alpha(x, y) g(x, y)$ is strongly pseudo-monotone.

Theorem 2. Suppose that the assumptions of Theorem 1 are satisfied. If any of the following conditions is satisfied,

i) $f$ is strongly pseudo-monotone,

ii) $f(x, \cdot)$ is strongly convex for all $x \in E$,

iii) $f(\cdot, y)$ is strongly concave for all $y \in E$,

then the sequence $\left\{x_{k}\right\}$ generated by the algorithm is strongly convergent to an element of $S(f, K)$.

Proof. Note that by Theorem 1, all cluster points of $\left\{x_{k}\right\}$ belong to $S(f, K)$. In the sequel, first in each item, we prove that if $x_{k_{i}} \rightarrow x^{*}$, then $x_{k_{i}} \rightarrow x^{*}$. Finally we prove that there is only one cluster point and hence $\left\{x_{k}\right\}$ converges strongly to a point of $S(f, K)$. Therefore we suppose that $x_{k_{i}} \rightarrow x^{*}$ and subsequently $z_{k_{i}} \rightarrow x^{*}$ by Lemma 1 . Note that, Lemma 1 implies that

$$
\lim _{i \rightarrow \infty}\left\|z_{k_{i}}-y_{k_{i}}\right\|=0 .
$$

Uniform smoothness of $E$ implies uniform norm-to-norm continuity of $J$ on each bounded set of $E$. Therefore, we get from (4.1),

$$
\lim _{i \rightarrow \infty}\left\|J z_{k_{i}}-J y_{k_{i}}\right\|=0
$$

Now, take $x^{*} \in S_{*}$, then by (3.2), we have $f\left(z_{k_{i}}, x^{*}\right)+\lambda_{k_{i}}\left\langle x^{*}-z_{k_{i}}, J z_{k_{i}}-J y_{k_{i}}\right\rangle \geq 0$, which implies that

$$
f\left(z_{k_{i}}, x^{*}\right)+\lambda_{k_{i}}\left\|x^{*}-z_{k_{i}}\right\|\left\|J z_{k_{i}}-J y_{k_{i}}\right\| \geq 0 .
$$

We use (4.2), (4.3) and the boundedness of $\left\{\lambda_{k}\right\}$ in order to obtain

$$
\liminf _{i \rightarrow \infty} f\left(z_{k_{i}}, x^{*}\right) \geq 0 \text {. }
$$

In the sequel, we continue to prove parts (i), (ii) and (iii) respectively:

i) Since $f\left(x^{*}, z_{k_{i}}\right) \geq 0$, by the definition of the strongly pseudo-monotone bifunction, there is $\beta>0$ such that $f\left(z_{k_{i}}, x^{*}\right) \leq-\beta\left\|z_{k_{i}}-x^{*}\right\|^{2}$. By taking liminf and using (4.4), we get

$$
0 \leq \liminf _{i \rightarrow \infty} f\left(z_{k_{i}}, x^{*}\right) \leq \liminf _{i \rightarrow \infty}\left(-\beta\left\|z_{k_{i}}-x^{*}\right\|^{2}\right) \leq-\beta \limsup _{i \rightarrow \infty}\left\|z_{k_{i}}-x^{*}\right\|^{2} .
$$

Therefore $z_{k_{i}} \rightarrow x^{*}$ and subsequently $x_{k_{i}} \rightarrow x^{*}$.

ii) By (3.2), we have $f\left(z_{k_{i}}, y\right)+\lambda_{k_{i}}\left\langle y-z_{k_{i}}, J z_{k_{i}}-J y_{k_{i}}\right\rangle \geq 0$. Now, let $\lambda \in(0,1)$ and set $p_{k_{i}}=\lambda z_{k_{i}}+(1-\lambda) x^{*}$, for all $i \in \mathbb{N}$. Since $f\left(z_{k_{i}}, \cdot\right)$ is strongly convex, we have

$$
\lambda_{k_{i}}\left\langle p_{k_{i}}-z_{k_{i}}, J y_{k_{i}}-J z_{k_{i}}\right\rangle \leq f\left(z_{k_{i}}, p_{k_{i}}\right)
$$




$$
\begin{aligned}
& \leq \lambda f\left(z_{k_{i}}, z_{k_{i}}\right)+(1-\lambda) f\left(z_{k_{i}}, x^{*}\right) \\
& -\lambda(1-\lambda)\left\|z_{k_{i}}-x^{*}\right\|^{2} \\
& \leq(1-\lambda) f\left(x_{k_{i}}, x^{*}\right)-\lambda(1-\lambda)\left\|z_{k_{i}}-x^{*}\right\|^{2} .
\end{aligned}
$$

Hence, we have

$$
\begin{aligned}
\lambda(1-\lambda)\left\|z_{k_{i}}-x^{*}\right\|^{2} & \leq \lambda_{k_{i}}\left\langle p_{k_{i}}-z_{k_{i}}, J z_{k_{i}}-J y_{k_{i}}\right\rangle+(1-\lambda) f\left(z_{k_{i}}, x^{*}\right) \\
& \leq \lambda_{k_{i}}\left\|p_{k_{i}}-z_{k_{i}}\right\|\left\|J z_{k_{i}}-J y_{k_{i}}\right\|+(1-\lambda) f\left(z_{k_{i}}, x^{*}\right) .
\end{aligned}
$$

Taking liminf in (4.5), we use (4.2) together with the boundedness of $\left\{z_{k_{i}}\right\}$ and $\left\{p_{k_{i}}\right\}$ in order to obtain that $\left\|z_{k_{i}}-x^{*}\right\| \rightarrow 0$. Therefore we have $x_{k_{i}} \rightarrow x^{*}$.

iii) Let $\lambda \in(0,1)$ and set $p_{k_{i}}=\lambda z_{k_{i}}+(1-\lambda) x^{*}$, for all $i \in \mathbb{N}$. Then since $f\left(\cdot, x^{*}\right)$ is strongly concave, we have

$$
\lambda f\left(z_{k_{i}}, x^{*}\right)+(1-\lambda) f\left(x^{*}, x^{*}\right)+\lambda(1-\lambda)\left\|z_{k_{i}}-x^{*}\right\|^{2} \leq f\left(p_{k_{i}}, x^{*}\right) \leq 0 .
$$

Therefore, we get $f\left(z_{k_{i}}, x^{*}\right) \leq-(1-\lambda)\left\|z_{k_{i}}-x^{*}\right\|^{2}$. Now, by taking liminf and (4.4), we get

$$
0 \leq \liminf _{i \rightarrow \infty} f\left(z_{k_{i}}, x^{*}\right) \leq-(1-\lambda) \limsup _{i \rightarrow \infty}\left\|z_{k_{i}}-x^{*}\right\|^{2} .
$$

Therefore $z_{k_{i}} \rightarrow x^{*}$. This implies that $x_{k_{i}} \rightarrow x^{*}$.

Now, we prove that there is only one cluster point and hence $\left\{x_{k}\right\}$ converges strongly to a point of $S(f, K)$. Note that we proved if $x_{k_{i}} \rightarrow x^{*}$, then $x_{k_{i}} \rightarrow x^{*}$. Now, if $\left\{x_{k_{i}}\right\}$ and $\left\{x_{k_{j}}\right\}$ are arbitrary subsequences of $\left\{x_{k}\right\}$ that converge strongly to $p$ and $q$, respectively, then

$$
\begin{aligned}
2\left\langle p-q, J x_{k_{i}}-J x_{k_{j}}\right\rangle & =2\left\langle p, J x_{k_{i}}\right\rangle-2\left\langle q, J x_{k_{i}}\right\rangle-2\left\langle p, J x_{k_{j}}\right\rangle+2\left\langle q, J x_{k_{j}}\right\rangle \\
& =-\phi\left(p, x_{k_{i}}\right)+\phi\left(q, x_{k_{i}}\right)+\phi\left(p, x_{k_{j}}\right)-\phi\left(q, x_{k_{j}}\right) .
\end{aligned}
$$

Since $\lim _{k \rightarrow \infty} \phi\left(p, x_{k}\right)$ and $\lim _{k \rightarrow \infty} \phi\left(q, x_{k}\right)$ exist by Lemma 1 and uniform smoothness of $E$ implies uniform (norm-to-norm) continuity of $J$ on each bounded set of $E$. Therefore, taking limit from the above equation when $i \rightarrow \infty$ and then when $j \rightarrow \infty$, we obtain $p=q$, i.e. $\left\{x_{k}\right\}$ converges strongly to a point of $S(f, K)$.

Theorem 3. Let $f$ be a bifunction and $K$ be a multivalued quasi $\phi$-nonexpansive mapping that the conditions B1-B6 are satisfied and that $\operatorname{int}\left(S_{*}\right) \neq \varnothing$. Then the sequence $\left\{x_{k}\right\}$ generated by the algorithm is strongly convergent to a solution of $Q E P(f, K)$.

Proof. Since $\operatorname{int}\left(S_{*}\right) \neq \varnothing$, there exist $r>0$ and $x^{*} \in \operatorname{int}\left(S_{*}\right)$ such that $\bar{B}_{r}\left(x^{*}\right) \subset$ $\operatorname{int}\left(S_{*}\right)$. Note that the duality mappings $J: E \rightarrow E^{*}$ and $J^{-1}: E^{*} \rightarrow E$ are single valued, one-to-one and surjective (see [12]). Now, if $\left\|J x_{k}-J y_{k}\right\| \neq 0$, replacing $\tilde{x}=x^{*}+r \frac{J^{-1}\left(J x_{k}-J y_{k}\right)}{\left\|J x_{k}-J y_{k}\right\|}$ by $x^{*}$ in (3.6), we have $\left\langle\tilde{x}-y_{k}, J x_{k}-J y_{k}\right\rangle \leq 0$ for all $k \in \mathbb{N}$, and 
hence

$$
\begin{aligned}
r\left\|J x_{k}-J y_{k}\right\| & =r\left\langle\frac{J^{-1}\left(J x_{k}-J y_{k}\right)}{\left\|J x_{k}-J y_{k}\right\|}, J x_{k}-J y_{k}\right\rangle \\
& \leq\left\langle x^{*}-y_{k}, J y_{k}-J x_{k}\right\rangle \\
& =\frac{1}{2}\left(\phi\left(x^{*}, x_{k}\right)-\phi\left(x^{*}, y_{k}\right)-\phi\left(y_{k}, x_{k}\right)\right) .
\end{aligned}
$$

Also if $\left\|J x_{k}-J y_{k}\right\|=0$, then (4.6) is satisfied. In the sequel, again if $\left\|J y_{k}-J z_{k}\right\| \neq 0$, replacing $\tilde{x}=x^{*}+r \frac{J^{-1}\left(J y_{k}-J z_{k}\right)}{\left\|J y_{k}-J z_{k}\right\|}$ by $x^{*}$ in (3.8), we have $\left\langle\tilde{x}-z_{k}, J y_{k}-J z_{k}\right\rangle \leq 0$ for all $k \in \mathbb{N}$, and hence

$$
\begin{aligned}
r\left\|J y_{k}-J z_{k}\right\| & =r\left\langle\frac{J^{-1}\left(J y_{k}-J z_{k}\right)}{\left\|J y_{k}-J z_{k}\right\|}, J y_{k}-J z_{k}\right\rangle \\
& \leq\left\langle x^{*}-z_{k}, J z_{k}-J y_{k}\right\rangle \\
& =\frac{1}{2}\left(\phi\left(x^{*}, y_{k}\right)-\phi\left(x^{*}, z_{k}\right)-\phi\left(z_{k}, y_{k}\right)\right) .
\end{aligned}
$$

Also if $\left\|J y_{k}-J z_{k}\right\|=0$, then (4.7) is satisfied. Once again, if $\left\|J z_{k}-J x_{k+1}\right\| \neq 0$, replacing $\tilde{x}=x^{*}+r \frac{J^{-1}\left(J x_{k}-J v^{k+1}\right)}{\left\|J x_{k}-J v^{k+1}\right\|}$ by $x^{*}$ in (3.10), we have $\left\langle\tilde{x}-x_{k+1}, J z_{k}-J x_{k+1}\right\rangle \leq 0$ for all $k \in \mathbb{N}$, and hence

$$
\begin{aligned}
r\left\|J z_{k}-J x_{k+1}\right\| & =r\left\langle\frac{J^{-1}\left(J z_{k}-J x_{k+1}\right)}{\left\|J z_{k}-J x_{k+1}\right\|}, J z_{k}-J x_{k+1}\right\rangle \\
& \leq\left\langle x^{*}-x_{k+1}, J x_{k+1}-J z_{k}\right\rangle \\
& =\frac{1}{2}\left(\phi\left(x^{*}, z_{k}\right)-\phi\left(x^{*}, x_{k+1}\right)-\phi\left(x_{k+1}, z_{k}\right)\right) .
\end{aligned}
$$

Also if $\left\|J z_{k}-J x_{k+1}\right\|=0$, then (4.8) is satisfied. Summing up (4.6), (4.7) and (4.8), we get

$$
\begin{aligned}
2 r\left\|J x_{k}-J x_{k+1}\right\| & \leq 2 r\left\|J x_{k}-J y_{k}\right\|+2 r\left\|J y_{k}-J z_{k}\right\|+2 r\left\|J z_{k}-J x_{k+1}\right\| \\
& \leq \phi\left(x^{*}, x_{k}\right)-\phi\left(x^{*}, x_{k+1}\right) .
\end{aligned}
$$

Summing up (4.9) from $k=0$ to $k=n$, we get

$$
2 r \sum_{k=0}^{n}\left\|J x_{k}-J x_{k+1}\right\| \leq \phi\left(x^{*}, x_{0}\right)-\phi\left(x^{*}, x_{n+1}\right) .
$$

Therefore we may conclude that $\sum_{k=0}^{\infty}\left\|J x_{k}-J x_{k+1}\right\|<\infty$. It follows that $\left\{J x_{k}\right\}$ converges strongly to an element in $E^{*}$. Since the duality mapping $J^{-1}$ is uniformly norm to norm continuous on each bounded subset of $E^{*}$, hence $\left\{x_{k}\right\}$ converges strongly to an element in $E$. On the other hand, since all cluster points of $\left\{x_{k}\right\}$ belong to $S(f, K)$ by Theorem 1, therefore $\left\{x_{k}\right\}$ converges strongly to an element of $S(f, K)$. 


\section{REFERENCES}

[1] Y. I. Alber, Metric and generalized projection operators in Banach spaces: properties and applications, ser. Lecture Notes in Pure and Appl. Math. Dekker, New York, 1996, vol. 178.

[2] D. Aussel, J. Cotrina, and A. N. Iusem, "An existence result for quasi-equilibrium problems," $J$. Convex Anal., vol. 24, no. 1, pp. 55-66, 2017.

[3] M. Bianchi and S. Schaible, "Generalized monotone bifunctions and equilibrium problems," $J$. Optim. Theory Appl., vol. 90, no. 1, pp. 31-43, 1996, doi: 10.1007/BF02192244. [Online]. Available: https://doi.org/10.1007/BF02192244

[4] O. Chadli, Z. Chbani, and H. Riahi, "Equilibrium problems with generalized monotone bifunctions and applications to variational inequalities," J. Optim. Theory Appl., vol. 105, no. 2, pp. 299-323, 2000, doi: 10.1023/A:1004657817758. [Online]. Available: https://doi.org/10.1023/A:1004657817758

[5] P. L. Combettes and S. A. Hirstoaga, "Equilibrium programming in Hilbert spaces," J. Nonlinear Convex Anal., vol. 6, no. 1, pp. 117-136, 2005.

[6] G. Z. Eskandani, M. Raeisi, and T. M. Rassias, "A hybrid extragradient method for solving pseudomonotone equilibrium problems using Bregman distance," J. Fixed Point Theory Appl., vol. 20, no. 3, pp. Paper No. 132, 27, 2018, doi: 10.1007/s11784-018-0611-9. [Online]. Available: https://doi.org/10.1007/s11784-018-0611-9

[7] A. N. Iusem, G. Kassay, and W. Sosa, "On certain conditions for the existence of solutions of equilibrium problems," Math. Program., vol. 116, no. 1-2, Ser. B, pp. 259-273, 2009, doi: 10.1007/s10107-007-0125-5. [Online]. Available: https://doi.org/10.1007/s10107-007-0125-5

[8] A. N. Iusem and M. Nasri, "Inexact proximal point methods for equilibrium problems in Banach spaces," Numer. Funct. Anal. Optim., vol. 28, no. 11-12, pp. 1279-1308, 2007, doi: 10.1080/01630560701766668. [Online]. Available: https://doi.org/10.1080/01630560701766668

[9] A. N. Iusem and W. Sosa, "Iterative algorithms for equilibrium problems," Optimization, vol. 52, no. 3, pp. 301-316, 2003, doi: 10.1080/0233193031000120039. [Online]. Available: https://doi.org/10.1080/0233193031000120039

[10] A. N. Iusem and W. Sosa, "On the proximal point method for equilibrium problems in Hilbert spaces," Optimization, vol. 59, no. 8, pp. 1259-1274, 2010, doi: 10.1080/02331931003603133. [Online]. Available: https://doi.org/10.1080/02331931003603133

[11] Z. Jouymandi and F. Moradlou, "Extragradient methods for solving equilibrium problems, variational inequalities, and fixed point problems," Numer. Funct. Anal. Optim., vol. 38, no. 11, pp. 1391-1409, 2017, doi: 10.1080/01630563.2017.1321017. [Online]. Available: https://doi.org/10.1080/01630563.2017.1321017

[12] S. Kamimura and W. Takahashi, "Strong convergence of a proximal-type algorithm in a Banach space," SIAM J. Optim., vol. 13, no. 3, pp. 938945 (2003), 2002, doi: 10.1137/S105262340139611X. [Online]. Available: https: //doi.org/10.1137/S105262340139611X

[13] H. Khatibzadeh and V. Mohebbi, "Proximal point algorithm for infinite pseudo-monotone bifunctions," Optimization, vol. 65, no. 8, pp. 1629-1639, 2016, doi: 10.1080/02331934.2016.1153639. [Online]. Available: https://doi.org/10.1080/02331934.2016.1153639

[14] H. Khatibzadeh and V. Mohebbi, "On the proximal point method for an infinite family of equilibrium problems in Banach spaces," Bull. Korean Math. Soc., vol. 56, no. 3, pp. 757-777, 2019, doi: 10.4134/BKMS.b180547. [Online]. Available: https: //doi.org/10.4134/BKMS.b180547

[15] H. Khatibzadeh, V. Mohebbi, and S. Ranjbar, "Convergence analysis of the proximal point algorithm for pseudo-monotone equilibrium problems," Optim. Methods Softw., vol. 30, 
no. 6, pp. 1146-1163, 2015, doi: 10.1080/10556788.2015.1025402. [Online]. Available: https://doi.org/10.1080/10556788.2015.1025402

[16] A. Moudafi, "Proximal point algorithm extended to equilibrium problems," J. Nat. Geom., vol. 15, no. 1-2, pp. 91-100, 1999.

[17] T. D. Quoc, P. N. Anh, and L. D. Muu, "Dual extragradient algorithms extended to equilibrium problems," J. Global Optim., vol. 52, no. 1, pp. 139-159, 2012, doi: 10.1007/s10898-011-9693-2. [Online]. Available: https://doi.org/10.1007/s10898-011-9693-2

[18] M. Raeisi and G. Z. Eskandani, "A hybrid extragradient method for a general split equality problem involving resolvents and pseudomonotone bifunctions in Banach spaces," Calcolo, vol. 56, no. 4, pp. Paper No. 43, 27, 2019, doi: 10.1007/s10092-019-0341-4. [Online]. Available: https://doi.org/10.1007/s10092-019-0341-4

[19] S. Reich, A weak convergence theorem for the alternating method with Bregman distances, ser. Lecture Notes in Pure and Appl. Math. Dekker, New York, 1996, vol. 178.

[20] N. T. T. Van, J. J. Strodiot, V. H. Nguyen, and P. T. Vuong, "An extragradienttype method for solving nonmonotone quasi-equilibrium problems," Optimization, vol. 67, no. 5, pp. 651-664, 2018, doi: 10.1080/02331934.2017.1416610. [Online]. Available: https://doi.org/10.1080/02331934.2017.1416610

Authors' addresses

Mehdi Mohammadi

Department of Mathematics, Payame Noor University, Tehran, Iran

E-mail address: mehdi.mohammadi5677@gmail.com

\section{G. Zamani Eskandani}

Department of Pure Mathematics, Faculty of Mathematical Sciences, University of Tabriz, Tabriz, Iran

E-mail address: zamani@tabrizu.ac.ir 